\title{
On the Cartesian product of an arbitrarily partitionable graph and a traceable graph
}

\author{
Olivier Baudon 1" ${ }^{1 *}$ \\ Antoni Marczyk ${ }^{2 \dagger}$ \\ Julien Bensmail ${ }^{1 *}$ \\ Jakub Przybyło ${ }^{2 \dagger}$ \\ ${ }^{1}$ University of Bordeaux and CNRS, LaBRI, 33405 Talence, France \\ ${ }^{2}$ AGH University of Science and Technology, 30-059 Krakow, Poland
}

Rafał Kalinowski \#

Mariusz Woźniak $^{2 \dagger}$

received 23rd Nov. 2012, accepted $26^{\text {th }}$ Mar. 2014.

\begin{abstract}
A graph $G$ of order $n$ is called arbitrarily partitionable (AP, for short) if, for every sequence $\tau=\left(n_{1}, \ldots, n_{k}\right)$ of positive integers that sum up to $n$, there exists a partition $\left(V_{1}, \ldots, V_{k}\right)$ of the vertex set $V(G)$ such that each set $V_{i}$ induces a connected subgraph of order $n_{i}$. A graph $G$ is called $\mathrm{AP}+1$ if, given a vertex $u \in V(G)$ and an index $q \in\{1, \ldots, k\}$, such a partition exists with $u \in V_{q}$. We consider the Cartesian product of AP graphs. We prove that if $G$ is $\mathrm{AP}+1$ and $H$ is traceable, then the Cartesian product $G \square H$ is $\mathrm{AP}+1$. We also prove that $G \square H$ is $\mathrm{AP}$, whenever $G$ and $H$ are AP and the order of one of them is not greater than four.
\end{abstract}

Keywords: partitions of graphs, Cartesian product of graphs, traceable graphs

\section{Introduction}

Let $G=(V, E)$ be a graph of order $n$, and $\tau=\left(n_{1}, \ldots, n_{k}\right)$ a sequence of positive integers. The sequence $\tau$ is admissible for $G$ if $n_{1}+\ldots+n_{k}=n$. Such an admissible sequence $\tau$ is said to be realizable in $G$ if we can partition $V$ into $k$ parts $\left(V_{1}, \ldots, V_{k}\right)$ such that $\left|V_{i}\right|=n_{i}$ and the subgraph $G\left[V_{i}\right]$ induced by $V_{i}$ is connected, for every $i=1, \ldots, k$. Note that in fact the ordering of $\tau$ is irrelevant, i.e., if $\tau$ is realizable in $G$, then it is also realizable after any permutation of its elements. We say that $G$ is arbitrarily partitionable (AP, for short) if every admissible sequence $\tau$ for $G$ is realizable in $G$. Furthermore, for a given integer $k \in\{1, \ldots, n\}$, a graph $G$ is called $k$-AP if every admissible sequence $\tau$ for $G$ of length at most $k$ is realizable in $G$. Note that every connected graph is 1-AP. Arbitrarily partitionable graphs, sometimes also called arbitrarily vertex decomposable, have been intensively studied for ten years (examples of papers are listed in the bibliography).

The notion of AP graphs was introduced by Barth et al. [1] (and independently by M. Horňák and M. Woźniak [10]) to model the following problem. Suppose that we own a connected network of $n$ resources,

*Emails: $\{$ olivier.baudon| julien.bensmail\}alabri.fr

†Emails: \{kalinows|marczyk|mwozniak\}@agh.edu.pl, przybylo@wms.mat.agh.edu.pl. The research was partially supported by the Polish Ministry of Science and Higher Education 
and we want to divide it among several customers. Every customer declares the number of resources that should be attributed to him. These numbers sum up to $n$. Moreover, each customer wants to receive a connected subnetwork of resources, and every resource must belong to exactly one subnetwork. Hence, we want our network to be partitionable as described, no matter how many subnetworks are requested (this number is at most $n$ ) and no matter what their orders are (but they sum up to $n$ ). Notice that any network with an underlying AP graph has this property.

Consider now an augmented version of this notion.

Let $\tau=\left(n_{1}, \ldots, n_{k}\right)$ be a sequence admissible for a graph $G$, let $\left(n_{i_{1}}, \ldots, n_{i_{r}}\right)$ be a subsequence of length $r$ of $\tau$, and let $\left\{v_{1}, v_{2}, \ldots, v_{r}\right\}$ be a set of $r$ vertices of $G$. The sequence $\tau$ is said to be realizable in $G$ under $r$ vertex restrictions $\left(v_{1}, n_{i_{1}}\right), \ldots,\left(v_{r}, n_{i_{r}}\right)$ if there exists a realization $\left(V_{1}, \ldots, V_{k}\right)$ of $\tau$ in $G$ such that $v_{p}$ belongs to a part $V_{i_{p}}$ for every $p=1, \ldots, r$. A graph $G$ is called arbitrarily partitionable under $r$ vertex restrictions (AP+r, for short) if, for each $r^{\prime}<r$, every admissible sequence for $G$ of length at least $r^{\prime}$ is realizable in $G$ under any $r^{\prime}$ vertex restrictions. Naturally, a graph is called $k-\mathrm{AP}+r$ if, for each $r^{\prime}<r$, every admissible sequence of length $l$, with $r^{\prime} \leq l \leq k$, is realizable in $G$ under $r^{\prime}$ vertex restrictions.

The problem of arbitrary partitioning graphs into connected subgraphs under vertex restrictions was already considered in late 70s by Lovász [13], and independently by Gyóri [7]. They both proved the following result.

Theorem 1 [13] A graph $G$ is $k$-connected if and only if $G$ is $k-A P+k$.

It is easily seen that each traceable graph is AP, so each condition implying the existence of a Hamiltonian path in a graph also implies that the graph is AP. Therefore, by weakening some known conditions for traceability we can expect to obtain sufficient conditions for being AP. For example, the well-known Ore's theorem states that if $G$ is a graph of order $n$ such that the degree sum of every pair of nonadjacent vertices is at least $n-1$, then $G$ is traceable. In [8] Horńák et al. showed that a connected graph $G$ of order $n \geq 20$ such that the degree sum of every pair of nonadjacent vertices is at least $n-5$ is AP if and only if it admits a perfect matching or a quasi-perfect matching (i.e., a matching omitting exactly one vertex). This is clearly an improvement of the above-mentioned Ore's condition for being AP.

Recall that given two graphs $G$ and $H$, the Cartesian product of $G$ and $H$, denoted by $G \square H$, is the graph whose vertex set is the Cartesian product $V(G) \times V(H)$ and whose two vertices $\left(u_{1}, v_{1}\right)$ and $\left(u_{2}, v_{2}\right)$ are adjacent if and only if either $u_{1}=u_{2}$ and $v_{1} v_{2} \in E(H)$, or $v_{1}=v_{2}$ and $u_{1} u_{2} \in E(G)$.

It is well known that if two graphs $G$ and $H$ are traceable then their Cartesian product $G \square H$ is also traceable. So one can formulate a conjecture.

Conjecture 2 If $G$ and $H$ are AP graphs, then the Cartesian product $G \square H$ is also AP.

Here we prove the following.

Theorem 3 The Cartesian product of two AP graphs is also AP, whenever at least one of these graphs is of order at most four.

Conjecture 2 seems to be very hard, so we formulate the following weaker conjecture by assuming traceability of $H$.

Conjecture 4 Let $G$ be an AP graph, and let $H$ be a traceable graph. Then $G \square H$ is AP. 
We also confirm this conjecture for small orders of $H$.

Theorem 5 If $G$ is an AP graph, then the Cartesian product $G \square H$ is AP, whenever $H$ is a traceable graph of order at most four.

It is easy to check that all AP graphs of order at most four are traceable (the smallest nontraceable AP graphs have order five). Hence, Theorem 3 is an immediate consequence of Theorem 5

We finally totally prove an AP+1 version of Conjecture 2.

Theorem 6 If $G$ is an $A P+1$ graph and $H$ is traceable, then the Cartesian product $G \square H$ is $A P+1$.

\section{Proof of Theorem 5}

If $a$ and $b$ are integers with $a<b$, then $[a, b]$ will denote the set of integers $\{a, a+1, \ldots, b-1, b\}$. Let $\tau=\left(n_{1}, \ldots, n_{k}\right)$ be any sequence. We will denote by $\|\tau\|$ the sum $n_{1}+\ldots+n_{k}$ and by $|\tau|$ the length $k$ of $\tau$. Let $\sigma$ be a permutation of the set $[1, k]$, and let $r \in[1, k-1]$. Then we say that $\tau$ is partitioned into two sequences $\tau^{1}=\left(n_{\sigma(1)}, \ldots, n_{\sigma(r)}\right)$ and $\tau^{2}=\left(n_{\sigma(r+1)}, \ldots, n_{\sigma(k)}\right)$, and we use the notation $\tau^{2}=\tau-\tau^{1}$ (here the sequence $\tau^{2}$ is determined up to the ordering of its elements). One can recursively extend this definition to a partition of $\tau$ into several sequences.

Clearly, it suffices to prove both Theorem 5 and Theorem 6 for $H$ being a path $P_{l}$ of order $l=|H|$. Thus, in both proofs, we focus on the Cartesian products of the form $G \square P_{l}$. Let $V\left(P_{l}\right)=\left\{p_{1}, \ldots, p_{l}\right\}$. The Cartesian product $G \square\left\{p_{i}\right\}$, where $i \in[1, l]$ and $\left\{p_{i}\right\}$ is identified with the graph $K_{1}$, is called the $i$-th layer of $G$ in $G \square P_{l}$ and denoted by $G^{i}$. For each $u \in V(G)$, the vertex $\left(u, p_{i}\right) \in V\left(G \square P_{l}\right)$ is called the $i$-th layer of $u$ in $G \square P_{l}$, and we will write $\left(u, p_{i}\right)=u^{i}$. Similarly, the $i$-th layer of a vertex subset $S \subseteq V(G)$ in $G \square P_{l}$, denoted by $S^{i}$, is the set of the $i$-th layers of all vertices of $S$.

The following simple lemma justifies the next proposition which is crucial in our proof of Theorem 5 .

Lemma 7 Let $l \geq 2$ be an integer, and $\tau=\left(n_{1}, \ldots, n_{k}\right)$ be a sequence of positive integers such that $\|\tau\| \equiv 0(\bmod l)$. If $k>l$, then $\tau$ can be partitioned into two nonempty subsequences $\tau^{1}$ and $\tau^{2}$ such that $\left\|\tau^{1}\right\| \equiv 0(\bmod l)$ and $\left\|\tau^{2}\right\| \equiv 0(\bmod l)$.

Proof: If $\tau$ contains an element $n_{i}$ such that $n_{i} \equiv 0(\bmod l)$, then if suffices to consider $\tau^{1}=\left(n_{i}\right)$ and $\tau^{2}=\tau-\left(n_{i}\right)$. Suppose then that $n_{i} \neq \equiv 0(\bmod l)$, for every $i \in[1, k]$.

For every $j \in[1, k]$, let $s_{j}=\sum_{i=1}^{j} n_{i}$ be the sum of the first $j$ elements of $\tau$. If there exist two indices $r$ and $t$ with $r<t$, such that $s_{r} \equiv s_{t}(\bmod l)$, then it is easy to see that $\tau^{1}=\left(n_{r+1}, \ldots, n_{t}\right)$ and $\tau^{2}=\tau-\tau^{1}$ satisfy our conditions. Since the number $k$ of elements of the sequence $s=\left(s_{1}, \ldots, s_{k}\right)$ is greater than $l$, the number of distinct residues modulo $l$, there must exist two elements of $s$ congruent modulo $l$. This ends the proof.

Proposition 8 Let $l \geq 2$ be a fixed integer such that for every connected graph $H$, the Cartesian product $H \square P_{l}$ is l-AP. Then $G \square P_{l}$ is AP for every AP graph $G$.

Proof: Let $G$ be an AP graph and $\tau$ a sequence admissible for $G \square P_{l}$. If $\tau$ has length at most 1 , then $\tau$ is realizable in $G \square P_{l}$ according to our assumption since $G$ is connected. Let us now suppose that $\tau$ has length at least $l+1$. By repeatedly applying Lemma 7, the sequence $\tau$ can be partitioned into subsequences $\tau^{1}, \ldots, \tau^{r}$ such that $\left\|\tau^{i}\right\| \equiv 0(\bmod l)$ and $\left|\tau^{i}\right| \leq l$ for every $i \in[1, r]$. 
For every $i \in[1, r]$, let $\alpha_{i}=\frac{\left\|\tau^{i}\right\|}{l}$. As $\left\|\tau^{i}\right\| \equiv 0(\bmod l)$, it follows that for each $i$, the number $\alpha_{i}$ is an integer. Clearly, the sequence $\alpha=\left(\alpha_{1}, \ldots, \alpha_{r}\right)$ sums up to the order of $G$ and hence, because $G$ is AP, admits a realization $\left(V_{1}, \ldots, V_{r}\right)$ in $G$. Put $U_{i}=V\left(G\left[V_{i}\right] \square P_{l}\right)$ for $i=1, \ldots, r$. Hence $\left(U_{1}, \ldots, U_{r}\right)$ is a partititon of the vertex set of $G \square P_{l}$, and each $U_{i}$ has exactly $\alpha_{i} l=\left\|\tau^{i}\right\|$ vertices. Since each subgraph $G\left[V_{i}\right]$ is connected, we infer by assumptions that each subgraph induced by $U_{i}$ admits a realization of $\tau^{i}$ within it. To obtain a global realization of $\tau$ in $G \square P_{l}$, we take realizations of all the sequences $\tau^{1}, \ldots, \tau^{r}$.

In view of Proposition 8 , to prove Conjecture 4 it suffices to show the following.

Conjecture 9 If $G$ is a connected graph, then $G \square P_{l}$ is $l$-AP for every integer $l \geq 1$.

In what follows, we prove that the assumptions of Proposition 8 hold for every $l \in[2,4]$. We will assume in this section that every sequence $\tau=\left(n_{1}, \ldots, n_{k}\right)$ is nonincreasing, i.e., $n_{1} \geq \cdots \geq n_{k}$.

Proposition 10 If $G$ is a connected graph and $l \geq 2$, then $G \square P_{l}$ is 2-AP.

Proof: If $G$ is connected, then clearly $G \square P_{l}$ is 2-connected. The conclusion follows from Theorem 1 .

Consequently, to prove that the Cartesian product of a connected graph $G$ and a path $P_{l}$ of order $l>2$ is $l$-AP, it suffices to consider sequences admissible for $G \square P_{l}$ which are of length greater than two.

Proposition 11 If $G$ is a connected graph, then $G \square P_{3}$ is 3-AP.

Proof: Suppose that the sequence $\tau=\left(n_{1}, n_{2}, n_{3}\right)$ is admissible for $G \square P_{3}$, with $n_{1}>n=|G|$ and $n_{3}<n$ (otherwise $n_{1}=n_{2}=n_{3}=n$ and $\left(V\left(G^{1}\right), V\left(G^{2}\right), V\left(G^{3}\right)\right)$ is an obvious realization of $\tau$ in $\left.G \square P_{3}\right)$. We distinguish two cases depending on the value of $n_{2}$.

- If $n>n_{2} \geq n_{3}$, let $T_{1}, T_{2} \subset V(G)$ be subsets of vertices such that $G\left[T_{1}\right]$ is a tree of order $n_{2}$, and $G\left[T_{2}\right]$ is a tree of order $n_{3}$. Observe then that the partition $\left(V\left(G \square P_{3}\right) \backslash\left(T_{1}^{1} \cup T_{2}^{3}\right), T_{1}^{1}, T_{2}^{3}\right)$ is a correct realization of $\tau$ in $G \square P_{3}$. Indeed, $G \square P_{3}-\left(T_{1}^{1} \cup T_{2}^{3}\right)$ is connected since every vertex from $V\left(G^{1}\right) \cup V\left(G^{3}\right) \backslash\left(T_{1}^{1} \cup T_{2}^{3}\right)$ has a neighbour in $V\left(G^{2}\right)$.

- If $2 n>n_{2} \geq n$, then $\left(V\left(G^{1}\right) \cup S_{1}, V\left(G^{3}\right) \cup S_{2}, T^{2}\right)$ is a realization of $\tau$, where $T \subset V(G)$ is chosen is such a way that $G[T]$ is a tree of order $n_{3}$, and $S_{1}$ and $S_{2}$ are subsets of vertices from $V\left(G^{2}\right) \backslash T^{2}$ chosen arbitrarily with $\left|S_{1}\right|=n_{1}-n$ and $\left|S_{2}\right|=n_{2}-n$. Notice that each vertex of $S_{1}$ or $S_{2}$ has a neighbour both in $V\left(G^{1}\right)$ and $V\left(G^{3}\right)$, so the subgraphs $\left(G \square P_{3}\right)\left[V\left(G^{1}\right) \cup S_{1}\right]$ and $\left(G \square P_{3}\right)\left[V\left(G^{3}\right) \cup S_{2}\right]$ are connected.

Proposition 12 If $G$ is a connected graph, then $G \square P_{4}$ is 4-AP.

\section{Proof:}

Suppose $\tau=\left(n_{1}, n_{2}, n_{3}\right)$ and $\|\tau\|=4 n$. We distinguish three cases.

- If $n>n_{2} \geq n_{3}$, then, for the same reasons as in Proposition $11 .\left(V\left(G \square P_{4}\right) \backslash\left(T_{1}^{1} \cup T_{2}^{4}\right), T_{1}^{1}, T_{2}^{4}\right)$ is a realization of $\tau$, where $T_{1}, T_{2} \subset V(G)$ are chosen in such a way that they induce trees of orders $n_{2}$ and $n_{3}$, respectively. 
- If $2 n>n_{2} \geq n>n_{3}$, then we can deduce a realization $\left(V_{1}, V_{2}, V_{3}\right)$ of $\left(n_{1}, n_{2}-n, n_{3}\right)$ in $\left(G \square P_{4}\right)\left[V\left(G^{2}\right) \cup V\left(G^{3}\right) \cup V\left(G^{4}\right)\right]$ using Proposition 11. Observe then that it can be extended to a realization of $\tau$ in $G \square P_{4}$ by simply considering $\left(V_{1}, V_{2} \cup V\left(G^{1}\right), V_{3}\right)$.

- $2 n>n_{2} \geq n_{3} \geq n$. Notice that $2 n \geq n_{1}$. Let $T_{2} \subseteq T_{1} \subset V(G)$ be subsets of vertices such that $G\left[T_{1}\right]$ and $G\left[T_{2}\right]$ are trees of orders $n_{2}-n$ and $n_{3}-n$, respectively. Consider now a sequence $\left(V\left(G^{1}\right) \cup\left(V\left(G^{2}\right) \backslash T_{1}^{2}\right), T_{1}^{2} \cup T_{2}^{3}, V\left(G^{4}\right) \cup\left(V\left(G^{3}\right) \backslash T_{2}^{3}\right)\right)$ of subsets of $V\left(G \square P_{4}\right)$. Notice that these three subsets induce connected subgraphs with the desired orders since every vertex of $V\left(G^{2}\right) \backslash T_{1}^{2}$ has a neighbour in $V\left(G^{1}\right)$, every one from $V\left(G^{3}\right) \backslash T_{2}^{3}$ is adjacent to a vertex in $V\left(G^{4}\right)$, and $T_{1}^{2}$ and $T_{2}^{3}$ have some adjacent vertices. Thus, $\left(V\left(G^{1}\right) \cup\left(V\left(G^{2}\right) \backslash T_{1}^{2}\right), T_{1}^{2} \cup T_{2}^{3}, V\left(G^{4}\right) \cup\left(V\left(G^{3}\right) \backslash T_{2}^{3}\right)\right)$ is a realization of $\tau$.

Now, consider the case where $\tau=\left(n_{1}, n_{2}, n_{3}, n_{4}\right)$, with $n_{1}>n$ and $n_{4}<n$ (otherwise $n_{1}=n_{2}=$ $n_{3}=n_{4}=n$, and $\left(V\left(G^{1}\right), V\left(G^{2}\right), V\left(G^{3}\right), V\left(G^{4}\right)\right)$ is a trivial realization of $\tau$ in $\left.G \square P_{4}\right)$. Depending on the values of $n_{2}$ and $n_{3}$, we claim that a realization of $\tau$ in $G \square P_{4}$ can always be found.

- $n>n_{2} \geq n_{3}$. Thus, $n_{1} \geq n$. Let $T_{3} \subseteq T_{2} \subseteq T_{1} \subset V(G)$ be subsets inducing subtrees of $G$ having orders $n_{4}, n_{3}$, and $n_{2}$, respectively. Notice then that $\left(V\left(G \square P_{4}\right) \backslash\left(T_{1}^{1} \cup T_{2}^{2} \cup T_{3}^{3}\right), T_{1}^{1}, T_{2}^{2}, T_{3}^{3}\right)$ is a realization of $\tau$ in $G \square P_{4}$ since every vertex from $V\left(G^{1}\right) \backslash T_{1}^{1}$ has a neighbour in $V\left(G_{2}\right) \backslash T_{2}^{2}$, every vertex from $V\left(G_{2}\right) \backslash T_{2}^{2}$ is adjacent to a vertex of $V\left(G_{3}\right) \backslash T_{3}^{3}$, and, finally, every vertex of $V\left(G_{3}\right) \backslash T_{3}^{3}$ has a neighbour in $V\left(G^{4}\right)$.

- $n_{2} \geq n>n_{3}$. Notice that $3 n>n_{1}+n_{3} \geq 2 n$ and $n<n_{2}+n_{4} \leq 2 n$. Let $T_{1}, T_{2} \subset V(G)$ be subsets inducing subtrees of $G$ of orders $n_{3}$ and $n_{4}$, respectively. Consider a sequence $\left(V\left(G^{2}\right) \cup\right.$ $\left.\left(V\left(G^{1}\right) \backslash T_{1}^{1}\right) \cup S_{1}, V\left(G^{4}\right) \cup S_{2}, T_{1}^{1}, T_{2}^{3}\right)$, where the subsets $S_{1}$ and $S_{2}$ are chosen arbitrarily from $V\left(G^{3}\right) \backslash T_{2}^{3}$ in such a way that $S_{1} \cap S_{2}=\emptyset,\left|S_{1}\right|=n_{1}+n_{3}-2 n$ and $\left|S_{2}\right|=n-\left|S_{1}\right|-n_{4}=n_{2}-n$. Observe that this is a realization of $\tau$ in $G \square P_{4}$ since every vertex of $\left(V\left(G^{1}\right) \backslash T_{1}^{1}\right) \cup\left(S_{1} \cup S_{2}\right)$ has a neighbour in $V\left(G_{2}\right)$ and all vertices of $S_{2}$ are adjacent to some vertices of $V\left(G^{4}\right)$.

- If $n_{2} \geq n_{3} \geq n$, let $T \subset V(G)$ be a subset of vertices inducing a subtree of $G$ with order $n_{4}$, $W \subseteq(V(G) \backslash T)$ be $n_{3}-n$ arbitrary vertices of $G$, and $u \in V(G)$ be a vertex of $G$ which is not an cut vertex. Consider now the realization $\left(V\left(G^{1}\right) \cup V\left(G^{2}\right) \cup V\left(G^{3}\right)\right) \backslash\left(S_{1} \cup S_{2} \cup T^{3} \cup\right.$ $\left.\left.W^{3}\right), S_{1} \cup S_{2}, V\left(G^{4}\right) \cup W^{3}, T^{3}\right)$ of $\tau$ in $G \square P_{4}$, where $S_{1}$ and $S_{2}$ are obtained in the following way. We start by putting $u^{1}$ and $u^{2}$ into $S_{1}$, and we start building a subtree of $V\left(G^{2}\right)$ rooted in $u^{2}$ iteratively, that is we take an arbitrary vertex $s^{2}$ joined to $V\left(G^{2}\right) \backslash S_{1}$ and add it to $S_{1}$. If $s^{3}$ does not belong to $W^{3}$, then $s^{3}$ is added to $S_{2}$. The procedure goes on until $\left|S_{1}\right|+\left|S_{2}\right| \geq n_{2}$. Observe that once the procedure is over, we can only have $\left|S_{1}\right|+\left|S_{2}\right| \in\left\{n_{2}, n_{2}+1\right\}$. In the case where $\left|S_{1}\right|+\left|S_{2}\right|=n_{2}+1$, we can remove the vertex $u^{1}$ from $S_{1}$ so that $S_{1} \cup S_{2}$ is a subset with size $n_{2}$. In any case, the four subgraphs induced by the realization are connected since $\left(G \square P_{4}\right)\left[S_{1}\right]$ induces a tree, every vertex from $S_{2}$ has a neighbour in $S_{1}$, and any vertex from $V\left(G^{3}\right) \backslash\left(S_{2} \cup T^{3} \cup W^{3}\right)$ is adjacent to one in $V\left(G^{2}\right) \backslash S_{2}$ which is itself adjacent to one in $V\left(G^{1}\right)$.

As a consequence of Propositions 8, 10, 11 and 12, we can state the following corollary, which in turn immediately implies Theorems 3 and 5

Corollary 13 If a graph $G$ is $A P$, and $l \leq 4$, then $G \square P_{l}$ is $A P$. 


\section{Proof of Theorem 6}

In this section, we also use the terminology and notation on the Cartesian product $G \square P_{l}$ which have we introduced in the initial paragraphs of Section 2. Clearly, it suffices to prove that $G \square P_{l}$ is $\mathrm{AP}+1$, with $l=|H|$.

Let $\tau=\left(n_{1}, \ldots, n_{k}\right)$ be a sequence which sums up to $n l$, the order of $G \square P_{l}$. Let $\left(u_{0}^{j}, n_{q}\right)$ be a vertex restriction. Without loss of generality, we may assume that $q=1$. In what follows, we explain how to find a realization of $\tau$ in $G \square P_{l}$ such that the $j$-th layer of a vertex $u_{0}$ in $G \square P_{l}$ belongs to a part of order $n_{1}$ in this realization.

Note that if $l=1$, then $G \square P_{1}$ is isomorphic to $G$, and thus it is $\mathrm{AP}+1$, by assumption. Hence, suppose that $l \geq 2$. We distinguish two cases depending on the cardinality of $n_{1}$. In each case, the proof goes in two stages. Roughly, we first modify the sequence $\tau$ by dividing some of its terms $n_{i}$ into two or more addends, so as $\tau$ could be partitioned into $l$ sequences $\tau^{1}, \ldots, \tau^{l}$ such that usually $\left\|\tau^{i}\right\|=n, i \in[1, l]$. Next, for all $i$, we construct a realization of $\tau^{i}$ in the $i$-th layer $G^{i}$ of $G \square P_{l}$ in such a way that parts whose sizes result from the splitting of a same element of $\tau$ yield a connected graph when glued together.

Case 1: $n_{1}<n$.

Consider the sequence $\tau^{\prime}=\left(n_{2}, \ldots, n_{k}\right)$. For each $i \in[1, l]$, we define a capacity $\varphi(i)$ of the $i$-th layer $G^{i}$ of $G \square P_{l}$ as follows:

$$
\varphi(i)= \begin{cases}n-n_{1} & \text { if } i=j, \\ n & \text { otherwise. }\end{cases}
$$

Now, we will recursively define $l$ sequences $\tau^{1}, \ldots, \tau^{l}$ such that every $\tau^{i}$ sums up to $\varphi(i)$, as follows. If there exists $p \in[2, k]$ such that $\sum_{i=2}^{p} n_{i}=\varphi(1)$, then we set $\tau^{1}=\left(n_{2}, \ldots, n_{p}\right), \tau^{\prime \prime}=\left(n_{p+1}, \ldots, n_{k}\right)$, and $\alpha(1)=0$ (for each $i \in[1, l-1]$, the number $\alpha(i)$ will indicate whether the last term of $\tau^{i}$ originated from dividing a term of $\tau$ ). Otherwise, let $p \in[2, k]$ be such that $\sum_{i=2}^{p-1} n_{i}\left\langle\varphi(1)\right.$ and $\sum_{i=2}^{p} n_{i}>$ $\varphi(1)$. Let $\eta_{1}=\varphi(1)-\sum_{i=2}^{p-1} n_{i}$ and $\eta_{2}^{\prime}=n_{p}-\eta_{1}$. We then set $\tau^{1}=\left(n_{2}, \ldots, n_{p-1}, \eta_{1}\right), \tau^{\prime \prime}=$ $\left(\eta_{2}^{\prime}, n_{p+1}, \ldots, n_{k}\right)$, and $\alpha(1)=1$.

We repeat this procedure with the sequence $\tau^{\prime \prime}$ instead of $\tau^{\prime}$ for the graph $G \square P_{l-1}$, where $P_{l-1}$ denotes the path $p_{2} \ldots p_{l}$, in order to obtain a sequence $\tau^{2}$ and the number $\alpha(2)$, and so on. After $l$ repetitions of this procedure, we obtain the desired sequences $\tau^{1}, \ldots, \tau^{l}$ such that $\left\|\tau^{i}\right\|=\varphi(i)$ for $i \in[1, l]$. Denote $\tau^{i}=\left(n_{1}^{i}, \ldots, n_{k_{i}}^{i}\right), i \in[1, l]$.

We begin our construction of a realization of $\tau$ by choosing a realization $\left(V_{1}, V_{1}^{j}, \ldots, V_{k_{j}}^{j}\right)$ in $G^{j}$ of the sequence $\left(n_{1}, n_{1}^{j}, \ldots, n_{k_{j}}^{j}\right)$ such that $u_{0}^{j} \in V_{1}$. We can choose it since this sequence sums up to $n$, and $G^{j}$ is $\mathrm{AP}+1$.

Next, for $i=j-1, j-2, \ldots, 1$, we proceed as follows. If $\alpha(i)=0$, then we take any realization $\left(V_{1}^{i}, \ldots, V_{k_{i}}^{i}\right)$ of $\tau^{i}$ in $G^{i}$. Otherwise, when $\alpha(i)=1$, we choose any vertex $u^{i+1} \in V_{1}^{i+1}$ and take a realization of $\tau^{i}$ in $G^{i}$ such that $u^{i} \in V_{k_{i}}^{i}$. This is possible since $G$ is AP+1. Note that the subgraph induced by $V_{k_{i}}^{i} \cup V_{1}^{i+1}$ in $G \square P_{l}$ is connected due to the edge $u^{i} u^{i+1}$ of the Cartesian product. Naturally, if $j=1$, then we omit this step and go directly to the step discribed below.

For every $i=j+1, \ldots, l$, if $\alpha(i-1)=0$, then we take any realization $\left(V_{1}^{i}, \ldots, V_{k_{i}}^{i}\right)$ of $\tau^{i}$ in $G^{i}$. Otherwise, when $\alpha(i-1)=1$, we choose a vertex $u^{i-1} \in V_{k_{i-1}}^{i-1}$, and take any realization of $\tau^{i}$ such that $u^{i} \in V_{1}^{i}$. Again, this is possible since $G$ is AP+1. Also the subgraph $\left(G \square P_{l}\right)\left[V_{k_{i-1}}^{i-1} \cup V_{1}^{i}\right]$ is connected. 
Thus, we obtained a realization

$$
\mathcal{R}=\left(V_{1}, V_{1}^{1}, \ldots, V_{k_{1}}^{1}, V_{1}^{2}, \ldots, V_{k_{l}}^{l}\right)
$$

of the sequence $\left(n_{1}, n_{1}^{1}, \ldots, n_{k_{1}}^{1}, n_{1}^{2}, \ldots, n_{k_{l}}^{l}\right)$. Suppose that in the procedure of defining the sequences $\tau^{1}, \ldots, \tau^{l}$, a certain term $n_{\nu}$ of $\tau$ has been divided into $s \geq 2$ addends $n_{k_{i}}^{i}, \ldots, n_{1}^{i+r-1}$. If $s \geq 3$, then of course, $k_{i+1}=\ldots=k_{i+s-2}=1$. Denote $V_{\nu}=V_{k_{i}}^{i} \cup V_{1}^{i+1} \cup \ldots \cup V_{1}^{i+s-1}$. By our construction, the subgraph $\left(G \square P_{l}\right)\left[V_{\nu}\right]$ is connected. We then replace the subsequence $V_{k_{i}}^{i}, V_{1}^{i+1}, \ldots, V_{1}^{i+s-1}$ in $\mathcal{R}$ by $V_{\nu}$. If we do the same for all divided terms of $\tau$, then we obtain a realization of $\tau$ in $G \square P_{l}$ satisfying the vertex restriction $\left(u_{0}^{j}, n_{1}\right)$.

Case 2: $n_{1} \geq n$.

Let $d=\left\lfloor\frac{n_{1}}{n-1}\right\rfloor$ and $r \equiv n_{1}(\bmod (n-1))$. In order to make our description more legible, we distinguish two subcases.

Subcase 2.1: $d<j$. We define the capacity function $\varphi$ for each $i$-th layer $G^{i}$ :

$$
\varphi(i)= \begin{cases}1 & \text { if } i \in[j-d+1, j], \\ n-r & \text { if } i=j-d, \\ n & \text { otherwise. }\end{cases}
$$

Then we consider the sequence $\tau^{\prime}=\left(n_{2}, \ldots, n_{k}\right)$, and we define the sequences $\tau^{1}, \ldots, \tau^{l}$, as well as numbers $\alpha(1), \ldots, \alpha(l)$, according to the function $\varphi$ exactly in the same way as in Case 1 .

Now, we start to construct a realization $\left(V_{1}, \ldots, V_{k}\right)$ of the sequence $\tau$ with choosing a vertex $x \in$ $V(G) \backslash\left\{u_{0}\right\}$, and defining the set $V_{1}$ of $n_{1}$ vertices as

$$
V_{1}=\bigcup_{i=j-d+1}^{j}\left(V\left(G^{i}\right) \backslash\left\{x^{i}\right\}\right) \cup W
$$

where $W$ is a set of $r$ vertices of $G^{j-d}$. When $r \neq 0$, we may additionally require, as $G$ is $\mathrm{AP}+1$, that $\left(W, V_{1}^{j-d}, \ldots, V_{k_{j-d}}^{j-d}\right)$ is a realization of the sequence $\left(r, n_{1}^{j-d}, \ldots, n_{k_{j-d}}^{j-d}\right)$ satisfying the vertex restriction $\left(x^{j-d}, n_{k_{j-d}}^{j-d}\right)$. When $r=0$, we consider any realization $\left(V_{1}^{j-d}, \ldots, V_{k_{j-d}}^{j-d}\right)$ of $\tau^{j-d}$, such that $x^{j-d} \in V_{k_{j-d}}^{j-d}$ if $\alpha(j-d-1)=1$.

Then, we consecutively produce realizations of $\tau^{j-d-2}, \tau^{j-d-3} \ldots, \tau^{1}$, following the same rules as in Case 1. That is, for every $i=j-d-2, \ldots, 1$, when $\alpha(i)=1$, we choose a vertex $u^{i+1} \in V_{1}^{i+1}$, and using the fact that $G$ is $\mathrm{AP}+1$, we find a realization of $\tau^{i}$ in $G^{i}$ such that $u^{i} \in V_{k_{i}}^{i}$. For $i \in[j-d+1, j]$, we take $\left\{x^{i}\right\}$ as a realization of the sequence $\tau^{i}=(1)$. Next, for $i=j+1, \ldots, l$, we proceed exactly in the same way as in Case 1.

The same arguments as in Case 1 show that we can glue corresponding elements of the realization $\left(V_{1}, V_{1}^{1}, \ldots, V_{k_{1}}^{1}, V_{1}^{2}, \ldots, V_{k_{l}}^{l}\right)$ of the sequence $\left(n_{1}, n_{1}^{1}, \ldots, n_{k_{1}}^{1}, n_{1}^{2}, \ldots, n_{k_{l}}^{l}\right)$ to obtain a realization $\left(V_{1}, \ldots, V_{k}\right)$ of $\tau$.

Subcase 2.2: $d \geq j$. If $n_{1}=n l$, then $k=1$ and $\tau=(n l)$ is trivially realizable in $G \square P_{l}$. For $k \geq 2$, we fix any vertex $x \in V(G) \backslash\left\{u_{0}\right\}$ and define

$$
V_{1}=\bigcup_{i=1}^{r} V\left(G^{i}\right) \cup \bigcup_{i=r+1}^{d}\left(V\left(G^{i}\right) \backslash\left\{x^{i}\right\}\right) .
$$


Clearly, $u_{0}^{j} \in V_{1}$. Then we consider the capacity function

$$
\varphi(i)= \begin{cases}1 & \text { if } i \in[r+1, d], \\ n & \text { if } i \in[d+1, n],\end{cases}
$$

and we define sequences $\tau^{r+1}, \ldots, \tau^{l}$ and their realizations according to the same rules as in Case 1 . It is easy to see that we can obtain a required realization $\left(V_{1}, \ldots, V_{k}\right)$ of $\tau$ in the same way as previously.

\section{References}

[1] D. Barth, O. Baudon and J. Puech, Network sharing: a polynomial algorithm for tripodes, Discrete Appl. Math. 119 (2002), 205-216.

[2] D. Barth, and H. Fournier: A degree bound on decomposable trees. Discrete Mathematics 306 (2006), 469-477.

[3] D. Barth, H. Fournier, and R. Ravaux: On the shape of decomposable trees. Discrete Math. 309 (2009), 3882-3887.

[4] O. Baudon, J. Bensmail, J. Przybyło, M. Woźniak, Partitioning powers of traceable or Hamiltonian graphs, submitted.

[5] O. Baudon, J. Przybyło, and M. Woźniak, On minimal arbitrarily partitionable graphs. Inf. Process. Lett. 112 (2012), 697-700.

[6] H.J. Broersma, D. Kratsch, and G.J. Woeginger, Fully decomposable split graphs, Lecture Notes in Computer Science 5874 (2009) 4105-112.

[7] E. Győri, On division of graphs to connected subgraphs, Combinatorics (Proc. Fifth Hungarian Colloq., Keszthely, 1976), Vol. I, pp. 485-494, Colloq. Math. Soc. János Bolyai, 18, North-Holland, Amsterdam-New York, 1978

[8] M. Horňák, A. Marczyk, I. Schiermeyer, and M. Woźniak, Dense arbitrarily vertex decomposable graphs, Graphs and Combin. 28 (2012), 807-821.

[9] M. Horňák, Zs. Tuza, and M. Woźniak, On-line arbitrarily vertex decomposable trees, Discrete Appl. Math. 155 (2007), 1420-1429.

[10] M. Horňák, and M. Woźniak, Arbitrarily vertex decomposable trees are of maximum degree at most six, Opuscula Mathematica 23 (2003), 49-62.

[11] R. Kalinowski, M. Pilśniak, M. Woźniak, and I.A. Zioło, Arbitrarily vertex decomposable suns with few rays, Discrete Math. 309 (2009), 3726-3732.

[12] R. Kalinowski, M. Pilśniak, M. Woźniak, and I.A. Zioło, On-line arbitrarily vertex decomposable suns, Discrete Math. 309 (2009), 6328-6336.

[13] L. Lovász, A homology theory for spanning trees of a graph, Acta Mathematica Academiae Scientiarum Hungaricae 30 (1977), 241-251.

[14] A.Marczyk, A note on arbitrarily vertex decomposable graphs, Opuscula Mathematica 26 (2006), 109-118.

[15] A. Marczyk, An ore-type condition for arbitrarily vertex decomposable graphs, Discrete Math. 309 (2009), 3588-3594. 